\title{
Very thin layers of T1P grown on InP using gas source molecular beam epitaxy
}

\author{
Jin-Shung Liu, Jyh-Shyang Wang, Hao-Hsiung Lin* \\ Department of Electrical Engineering, Room 419, National Taiwan University, Taipei, Taiwan, ROC
}

Received 16 March 1998

\begin{abstract}
Growth of TlP on InP substrates was attempted using gas source molecular beam epitaxy. When the growth temperature is lower than $420^{\circ} \mathrm{C}, \mathrm{Tl}_{3} \mathrm{P}$ droplets were found on the InP surface. For higher growth temperatures, a thin accumulation $\mathrm{Tl}$ layer in the surface region of the sample was detected by secondary ion mass spectroscopy. By increasing the $\mathrm{Tl}$ flux to $8 \mathrm{ML} / \mathrm{s}$ at a growth temperature of $450^{\circ} \mathrm{C}$, besides the enhancement on the surface density of the $\mathrm{Tl}$ layer, an additional peak in the double crystal X-ray rocking curve was found. The $8.5 \mathrm{~K}$ photoluminescence spectra are also presented. (C) 1998 Elsevier Science B.V. All rights reserved.
\end{abstract}

PACS: $68.55 . \mathrm{Bd} ; 68.55 . \mathrm{Jk} ; 68.55 . \mathrm{Nq}$

Keywords: Gas source molecular beam epitaxy; III-V compound semiconductors

\section{Introduction}

Mid- and long-wave infrared detectors and lasers are important optoelectronic devices. Recently, a new infrared material system, InT1P, was proposed [1] as an alternative of the dominant $\mathrm{InSb}$ and $\mathrm{HgCdTe}$ alloys for such applications. The theoretical calculations predicted that TlP is a semimetal with a band gap of $-0.27 \mathrm{eV}$ and therefore, $\operatorname{In}_{1-x} \mathrm{Tl}_{x} \mathrm{P}$ spans almost the entire infrared spectrum. Also, it was suggested that $\mathrm{In}_{1-x} \mathrm{Tl}_{x} \mathrm{P}$ can be grown in the zinc-blende

\footnotetext{
*Corresponding author: Fax: + 8862 3632442; e-mail: hhlin@cc.ee.ntu.edu.tw.
}

structure for all compositions with its lattice constant nearly matching that of InP. Few reports have been published regarding the growth of these materials [2-4]. Among them, only Asahi et al. claimed the successful growth of T1P and InT1P using gas source molecular beam epitaxy (GSMBE). They reported double crystal X-ray diffraction (DXRD) rocking curves for T1P and InT1P on InP substrates and photoluminescence (PL) spectrum for InT1P with only $1 \% \mathrm{Tl}$ composition $[3,4]$. In this study, besides the similar DXRD rocking curves and PL spectra, the results of secondary ion mass spectroscopy (SIMS) and energy dispersive spectroscopy (EDS) are reported. When the growth temperature is lower than $420^{\circ} \mathrm{C}, \mathrm{Tl}_{3} \mathrm{P}$ droplets were found on the InP surface. For higher growth temperatures, 
a thin $\mathrm{Tl}$ accumulation layer in InP, but not in the T1P layer, was detected by SIMS.

\section{Experimental procedure}

The growth of T1P on Fe-doped semi-insulating (1 100$)$ InP substrates was attempted using VG V80H GSMBE. Elemental Tl and In were used as the group III sources and thermally cracked $\mathrm{PH}_{3}$ was used as the group V source. The amount of $\mathrm{Tl}$ input beam flux was estimated from the comparison with the calibrated In beam flux by the conventional beam equivalent pressure (BEP) method, where the different ionization efficiencies for In and $\mathrm{Tl}$ in the ion gauge were taken into account. The dependence of $\mathrm{Tl}$ vapor pressure on the cell temperature is similar to the reported results $[2,4]$.

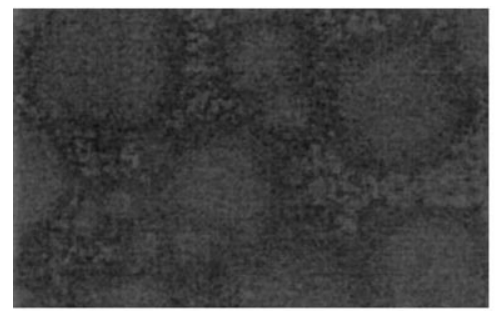

(a)

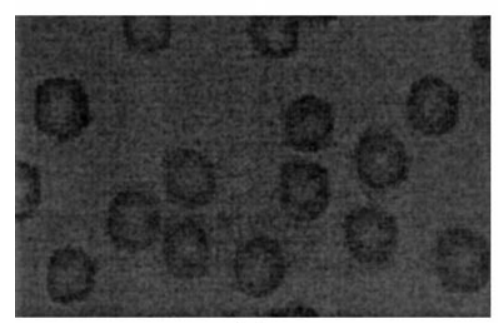

(c)

\section{Results and discussion}

A set of samples with relatively lower growth temperatures ranging from 350 to $420^{\circ} \mathrm{C}$ was first attempted. The $\mathrm{Tl}$ and $\mathrm{PH}_{3}$ input fluxes were kept at about 1 and $6 \mathrm{ML} / \mathrm{s}$, respectively. After the growth, a 300-^-thick InP cap was overgrown at the same growth temperature. The surface morphologies of the grown samples are shown in Fig. 1. It can be seen that the surface of sample grown at $420^{\circ} \mathrm{C}$ is mirror-like but the others contain many large droplets with the density increasing with the decrease of growth temperature. EDS was used to analyze these samples. No Tl signal can be detected for the sample grown at $420^{\circ} \mathrm{C}$ and for the dropletfree regions of the samples grown below $420^{\circ} \mathrm{C}$. Alternatively, the droplet is mainly composed of $\mathrm{Tl}$ and $\mathrm{P}$ with the $\mathrm{Tl} / \mathrm{P}$ composition ratio about $3-4$ in

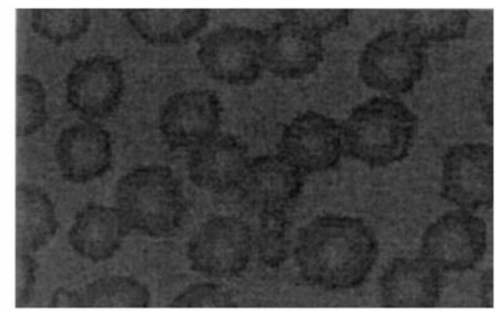

(b)

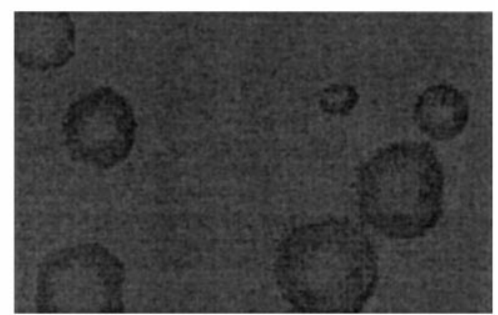

(d)

$20 \mu \mathrm{m}$

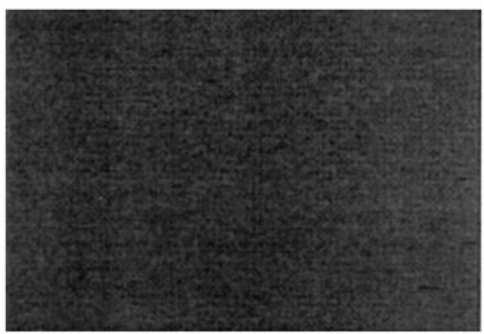

(e)

Fig. 1. The surface morphologies of samples grown at (a) $350^{\circ} \mathrm{C}$, (b) $370^{\circ} \mathrm{C}$, (c) $385^{\circ} \mathrm{C}$, (d) $400^{\circ} \mathrm{C}$, and (e) $420^{\circ} \mathrm{C}$. 
all samples. These findings show that a growth temperature of $420^{\circ} \mathrm{C}$ was high enough to re-evaporate the incident $\mathrm{Tl}$ atoms from the growing surface. On the other hand, the lower growth temperature improves the sticking coefficient of $\mathrm{Tl}$. However, instead of two-dimensional growth, the resident $\mathrm{Tl}$ adatoms form droplets with $\mathrm{P}$ adatoms. The composition is $\mathrm{Tl}_{3} \mathrm{P}$, according to the EDS results. This suggests that the formation of a twodimensional TIP epilayer is not thermodynamically preferable at growth temperatures lower than $420^{\circ} \mathrm{C}$.

The second set of samples was grown at relatively higher temperatures. The growth temperatures were 420,435 and $450^{\circ} \mathrm{C}$, respectively. For the former two samples, which were grown at 420 and $435^{\circ} \mathrm{C}$, respectively, the input $\mathrm{Tl}$ and $\mathrm{PH}_{3}$ fluxes were adjusted to 0.5 and $9 \mathrm{ML} / \mathrm{s}$, respective1y. This very high $\mathrm{Tl} / \mathrm{P}$ ratio of 18 was an attempt to suppress the $\mathrm{Tl}$ desorption. On the other hand, with the same $\mathrm{PH}_{3}$ flux of $9 \mathrm{ML} / \mathrm{s}$, the input Tl flux for the sample grown at $450^{\circ} \mathrm{C}$ was further increased to be as high as $8 \mathrm{ML} / \mathrm{s}$. The use of high input $\mathrm{Tl}$ flux was attempted to increase the total amount of $\mathrm{Tl}$ incorporation atoms. After the growth, a $0.4-\mu \mathrm{m}$-thick InP cap was overgrown at the same growth temperature for each sample in this set.

The samples grown at 420 and $435^{\circ} \mathrm{C}$ have mirror-like surfaces and still contain no $\mathrm{Tl}$ atoms according to the EDS results. It indicates that a high $\mathrm{Tl} / \mathrm{P}$ ratio still cannot effectively prevent the $\mathrm{Tl}$ desorption. On the other hand, the surface of the sample grown at $450^{\circ} \mathrm{C}$ has a high density of tiny droplets and some larger droplets with tails, as shown in Fig. 2. The most remarkable finding is that unlike all the other previous grown samples for which only InP substrate peaks were observed in their DCXD rocking curves, this sample has an additional diffraction peak as can be seen in Fig. 3 . The splitting angle between the additional peak and InP peaks is about 105 arcsec. This value is smaller than the reported value of 278 arcsec for TIP by Asahi et al. To further analyze the epilayer, SIMS was employed, and the SIMS profiling is shown in Fig. 4. The striking finding is that the expected T1P epilayer does not exist in this sample. As can be seen, $\mathrm{Tl}$ accumulates only in the surface region and decays very fast in the epilayer. Note that the accumulation layer is in the cap InP layer. Such a profile clearly indicates the surface segregation of $\mathrm{Tl}$ during the growth of both the nominal TIP layer and the following InP cap layer. The SIMS profiles of the samples grown at 420 and $435^{\circ} \mathrm{C}$ also show similar results. However, their surface counts are about one-hundredth of that of the sample grown at $450^{\circ} \mathrm{C}$, and are beyond the detectivity of the EDS.

To confirm the finding from SIMS profiling, the $0.3-\mu \mathrm{m}$-thick cap layer of the sample grown at $450^{\circ} \mathrm{C}$ was etched away. The DCXD rocking curve

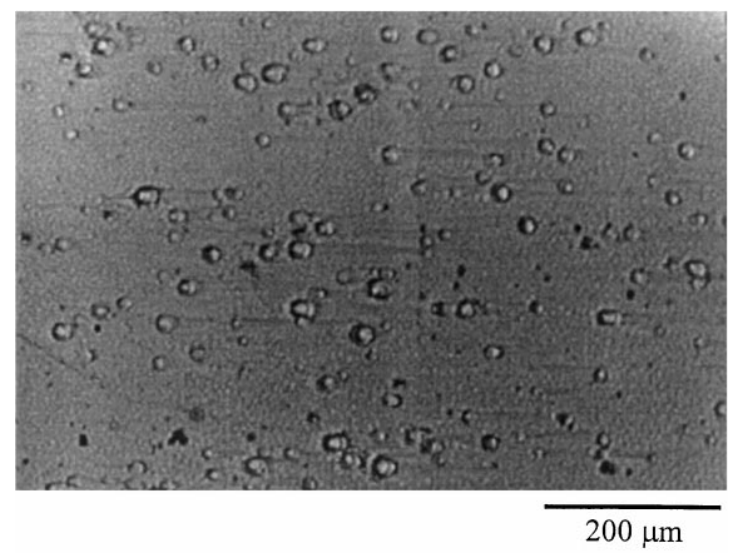

Fig. 2. The surface morphology of the sample grown at $450^{\circ} \mathrm{C}$.

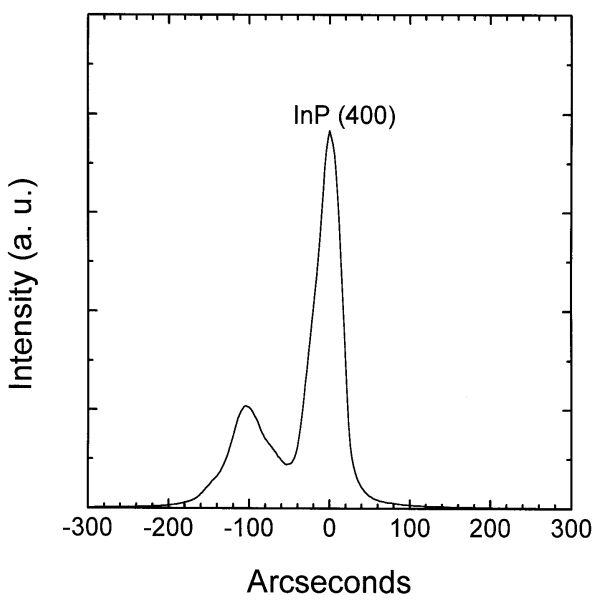

Fig. 3. The (4 0 0)DCXD rocking curve of the sample grown at $450^{\circ} \mathrm{C}$. 


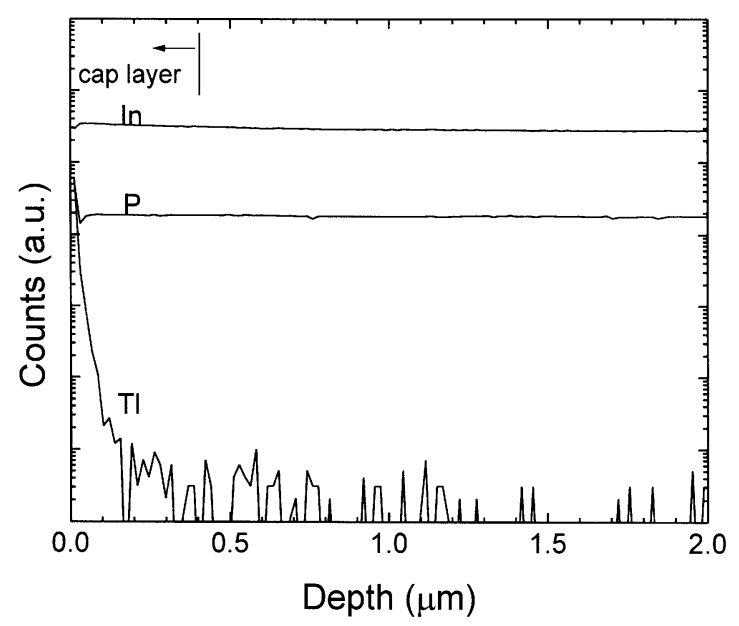

Fig. 4. The SIMS profiles of the sample grown at $450^{\circ} \mathrm{C}$.

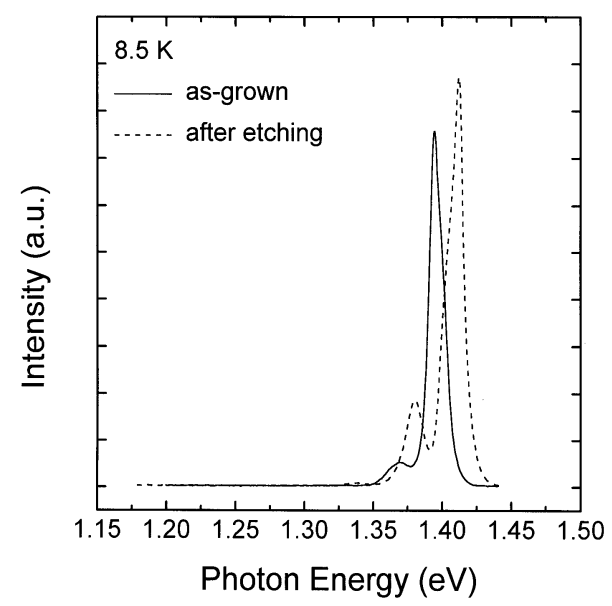

Fig. 5. The $8.5 \mathrm{~K} \mathrm{PL}$ spectra of the sample grown at $450^{\circ} \mathrm{C}$.

of the etched sample was measured again. It was found that the additional diffraction peak disappears. The $8.5 \mathrm{~K}$ PL spectra for the as-grown and etched samples are shown in Fig. 5. As can be seen, the spectrum for the as-grown sample exhibits a red-shift of about $20 \mathrm{meV}$ compared with that of the etched sample, which is similar to that of undoped InP grown in our system. Clearly, the red-shift results from the incorporation of $\mathrm{Tl}$ atoms accumulated in the surface region.
Based on these results, only a low $\mathrm{Tl}$ content of InT1P surface layer exists in the sample. Because of no standard sample, our SIMS measurement could not provide absolute $\mathrm{Tl}$ content. A rough estimation can be made from the EDS measurement that shows the $\mathrm{Tl}$ content to be $0.17 \%$. This result was used to convert the integrated SIMS counts ratio between In and $\mathrm{Tl}$ within the $1 \mu \mathrm{m}$ spot size of the EDS probe to estimate on absolute concentration. The derived surface $\mathrm{Tl}$ mole fraction ratio is about 0.2 , which corresponds to a lattice-mismatch between TlP and InP of $0.4 \%$. However, our result is only a rough estimation; further measurements are needed.

\section{Conclusions}

In summary, a series of samples were attempted for the growth of T1P on InP substrates using GSMBE. When the growth temperature is lower than $420^{\circ} \mathrm{C}, \mathrm{Tl}_{3} \mathrm{P}$ droplets were found on the InP surface. For higher growth temperatures, a thin accumulation $\mathrm{Tl}$ layer in the surface region of the sample was detected by SIMS. By increasing the Tl flux to $8 \mathrm{ML} / \mathrm{s}$, a thin accumulation Tl layer in the surface region of the sample grown at $450^{\circ} \mathrm{C}$ was found by using SIMS. DXRD and $8.5 \mathrm{~K}$ PL of the layer are presented. A rough estimation suggests that the lattice mismatch between TIP and InP is about $0.4 \%$.

\section{Acknowledgements}

This work was supported by the National Science Council, R.O.C. (NSC 87-2215-E-002-010).

\section{References}

[1] M.V. Schilfgaarde, A.B. Chen, S. Krishnamurthy, A. Sher, Appl. Phys. Lett. 65 (1994) 2714.

[2] M.J. Anotonell, Mater. Res. Soc. Symp. Proc. (1995) 379.

[3] H. Asahi, K. Yamamoto, K. Iwata, S. Gonda, K. Oc, Jpn. J. Appl. Phys. 35 (1996) L876.

[4] K. Yamamoto, H. Asahi, M. Fushida, K. Iwata, S. Gonda, J. Appl. Phys. 81 (1997) 1704. 